\title{
Suppression of MAL gene expression is associated with colorectal cancer metastasis
}

\author{
RONGQIANG MA 1* $^{*}$ YE XU ${ }^{2 *}$, MING WANG ${ }^{2}$ and WEI PENG ${ }^{2}$ \\ ${ }^{1}$ Department of Digestive Surgery, Xijing Hospital of Digestive Diseases, The Fourth Military Medical University, Xi'an, \\ Shaanxi 710000; ${ }^{2}$ Department of Digestive Surgery, Shanghai Traditional Chinese Medicine-Integrated Hospital, \\ Shanghai 200082, P.R. China
}

Received July 24, 2014; Accepted April 8, 2015

DOI: $10.3892 / \mathrm{ol} .2015 .3355$

\begin{abstract}
Mal, T-cell differentiation protein (MAL) is a candidate tumor suppressor gene that functions in membrane trafficking processes in polarized epithelial cells. The aim of the present study was to determine its clinical significance in colorectal cancer (CRC). The RNA and protein expression levels of MAL in 30 colorectal specimens were detected by semi-quantitative polymerase chain reaction and immunohistochemistry analysis. Statistical analysis was performed using SPSS software. The RNA level of MAL was significantly downregulated in the CRC tissues compared with the adjacent healthy tissue $(\mathrm{P}<0.05)$. MAL was only positively expressed in $20 \%$ of the CRC tissues, but in $66.7 \%$ of the adjacent tissues, as determined by immunohistochemistry analysis. The expression of the MAL RNA transcript exhibited a positive correlation with protein expression. The expression levels of MAL were significantly associated with different tumor-node-metastasis stages and lymph node metastasis $(\mathrm{P}<0.05)$, but not with age, gender, tumor site, differentiation status and pathological type (P>0.05). Suppression of MAL expression was significantly correlated with metastasis in CRC. The present study indicated that MAL may function as an anti-metastasis factor and represent a potential biomarker for malignant colorectal tumors.
\end{abstract}

\section{Introduction}

Colorectal cancer (CRC) is the third most common malignant cancer and the second leading cause of mortality worldwide (1). In China, the incidence rate of CRC is increasing rapidly, particular in urban areas, such as Shanghai, which was formerly considered a low-risk area $(2,3)$. Metastasis is

Correspondence to: Dr Wei Peng, Department of Digestive Surgery, Shanghai Traditional Chinese Medicine-Integrated Hospital, 230 Baoding Road, Houkou, Shanghai 200082, P.R. China E-mail: pengwei2012@126.com

*Contributed equally

Key words: colorectal cancer, MAL, metastasis the main cause of mortality in CRC patients (4). The first sites of metastatic $\mathrm{CRC}$ are the regional lymph nodes and the liver. Investigations into the molecular mechanisms involved in CRC metastasis are of major importance in order to develop novel strategies for targeted therapies (5).

The mal, T-cell differentiation protein (MAL) gene encodes the T-lymphocyte maturation-associated protein and functions in T-cell differentiation (6). Recently, downregulation of MAL has been associated with a variety of human epithelial malignancies. For example, Mimori et al (7) initially found that MAL was highly expressed in normal esophageal epithelia, but silenced in esophageal tumors. Later, inactivation of MAL was shown to be a common event in breast, head and neck, and gastric cancer (8-11). However, the functional role and mechanistic action of MAL in CRC remains largely unknown. The present study aimed to determine the clinical significance of MAL in colorectal cancer by measuring its expression level in CRC tissues.

\section{Materials and methods}

Tissue samples and cell line. The present study enrolled a total of 30 CRC patients who underwent surgery between 2010 and 2011 at Shanghai First People's Hospital (Shanghai, China). The patients did not receive any pre-operative cancer treatment prior to resection. Of the 30 patients, 18 were male and 22 were female, and the age of the patients ranged between 45 and 87 years (median, 69 years). The clinicopathological data of the CRC patients are shown in Table I.

Ethical approval. All clinical tissues were collected from the CRC patients after obtaining informed consent according to an established protocol approved by the Ethics Committee of Shanghai First People's Hospital.

RNA isolation and semi-quantitative polymerase chain reaction $(P C R)$. Total RNA was extracted from CRC patient samples using the SQ DNA/RNA/Protein Tissue kit (\#R8042-01; Sangon Biotech Co., Ltd., Shanghai, China) and used for cDNA synthesis with the Takara RNA PCR kit (AMV) Ver.3.0 (\#RR019; Takara Biotechnology Co., Ltd., Dalian, China). MAL and $\beta$-actin were detected, and their primers were as follows: MAL forward, 
Table I. Clinical chracteristics of the colorectal cancer patients.

\begin{tabular}{|c|c|c|c|c|c|c|c|}
\hline Case & Gender & Age, years & Grade & Specimen site & TNM & Stage & Type \\
\hline 1 & $\mathrm{~F}$ & 67 & G1 & Right & T4aN1aM0 & IIIB & 2 \\
\hline 2 & M & 66 & G1 & Left & T4aN0M0 & IIB & 1 \\
\hline 3 & $\mathrm{~F}$ & 73 & G3 & Left & T4aN1bM0 & IIIB & 1 \\
\hline 4 & M & 72 & $\mathrm{G} 2$ & Left & T4aN1bM0 & IIIB & 1 \\
\hline 5 & M & 77 & $\mathrm{G} 2$ & Right & T4aN0M0 & IIIB & 1 \\
\hline 6 & M & 62 & G2 & Left & T4aNOM0 & IIB & 1 \\
\hline 7 & M & 69 & G2 & Right & T4aN0M0 & IIB & 1 \\
\hline 8 & M & 62 & G2 & Left & T4aN1aM0 & IIIB & 1 \\
\hline 9 & $\mathrm{~F}$ & 77 & G2 & Rectum & T4aN0M0 & IIB & 1 \\
\hline 10 & M & 86 & G2 & Rectum & T2N0M0 & I & 1 \\
\hline 11 & $\mathrm{~F}$ & 64 & $\mathrm{G} 2$ & Rectum & T4aN1bM0 & IIIB & 1 \\
\hline 12 & $\mathrm{~F}$ & 77 & $\mathrm{G} 2$ & Left & T2N0M0 & I & 1 \\
\hline 13 & $\mathrm{~F}$ & 75 & G1 & Rectum & T1N0M0 & I & 1 \\
\hline 14 & M & 69 & $\mathrm{G} 2$ & Right & T4aNOM0 & IIB & 1 \\
\hline 15 & $\mathrm{~F}$ & 73 & G3 & Right & T4aN2bM0 & IIIC & 1 \\
\hline 16 & M & 69 & G1 & Rectum & T2N1aM0 & IIIA & 2 \\
\hline 17 & M & 53 & $\mathrm{G} 2$ & Rectum & T4aN0M0 & IIB & 1 \\
\hline 18 & M & 70 & G1 & Left & T4aNOM0 & IIB & 1 \\
\hline 19 & $\mathrm{~F}$ & 55 & G2 & Right & T4aN1bM0 & IIIB & 1 \\
\hline 20 & M & 75 & $\mathrm{G} 2$ & Left & T2N0M0 & I & 1 \\
\hline 21 & M & 45 & G2 & Rectum & T4aNOM0 & IIB & 2 \\
\hline 22 & M & 87 & G2 & Right & T4aNOM0 & IIB & 1 \\
\hline 23 & $\mathrm{~F}$ & 78 & G2 & Right & T2N0M0 & I & 1 \\
\hline 24 & $\mathrm{~F}$ & 51 & G2 & Right & T4aNOM0 & IIB & 1 \\
\hline 25 & M & 78 & G1 & Rectum & T4aNOM0 & IIB & 1 \\
\hline 26 & $\mathrm{~F}$ & 61 & G3 & Left & T4aN1bM0 & IIIB & 1 \\
\hline 27 & M & 63 & G2 & Rectum & $\mathrm{T} 2 \mathrm{~N} 2 \mathrm{bM} 0$ & IIIB & 1 \\
\hline 28 & $\mathrm{~F}$ & 59 & G2 & Right & T4aN1M0 & IIIB & 1 \\
\hline 29 & M & 75 & G2 & Rectum & T4aNOM0 & IIB & 1 \\
\hline 30 & M & 72 & G2 & Rectum & T4aN1M0 & IIIB & 1 \\
\hline
\end{tabular}

TNM, tumor-node-metastasis. The staging system used was based on the American Joint Committee on Cancer TNM system (12).

5'-TGGGTGATGTTCGTGTCTGTG-3'; and reverse, 5'-TCAAGTTCTACTGCGGCTTTATG-3'; and $\beta$-actin forward, 5'-CTGGGACGACATGGAGAAAA-3' and reverse, 5'-AAGGAAGGCTGGAAGAGTGC-3'.

Immunohistochemical staining. Immunohistochemical staining was performed to detect the expression of MAL in the CRC and matched non-cancer tissues. The primary antibody against MAL was obtained from Santa Cruz Biotechnology Inc. (\#sc-66978; Dallas, TX, USA). The intensity of staining was scored as follows: 0 , negative; 1 , weak; 2 , moderate; or 3 , strong. The extent of staining was based on the percentage of positive tumor cells: 0 , negative; $1,1-25 \% ; 2,26-50 \%$; $3,51-75 \%$; and $4,76-100 \%$. The final score of each sample was assessed by the sum of the results of the intensity and extent of staining. Therefore, each case was considered negative if the final score was $0-1(-)$ or 2-3 ( \pm ), and positive if the final score was 4-5 (+) or 6-7 (++), respectively. These scores were determined independently by two senior pathologists.
Statistical analysis. Statistical analysis was performed using SPSS 16.0 (SPSS, Inc., Chicago, IL, USA) and values are expressed as the mean \pm standard deviation. The $\chi^{2}$-test was used to evaluate the differences in staining of MAL according to the patient and tumor characteristics. The differences between groups were analyzed by using Student's t-test. Spearman's rank test was used for analysis of correlations between MAL RNA and protein levels. $\mathrm{P}<0.05$ was considered to indicate a statistically significant difference.

\section{Results}

RNA expression level of MAL in CRC and adjacent tissues. By performing a semi-quantitative PCR assay, MAL RNA expression was detected in the $30 \mathrm{CRC}$ tissues and the adjacent non-cancer tissues. The expression of $\beta$-actin was considered as the endogenous control. A total of 9 CRC specimens showed expression of MAL, with a positive rate of $30 \%(9 / 30)$, and 23 adjacent tissues showed expression of MAL, with a positive 

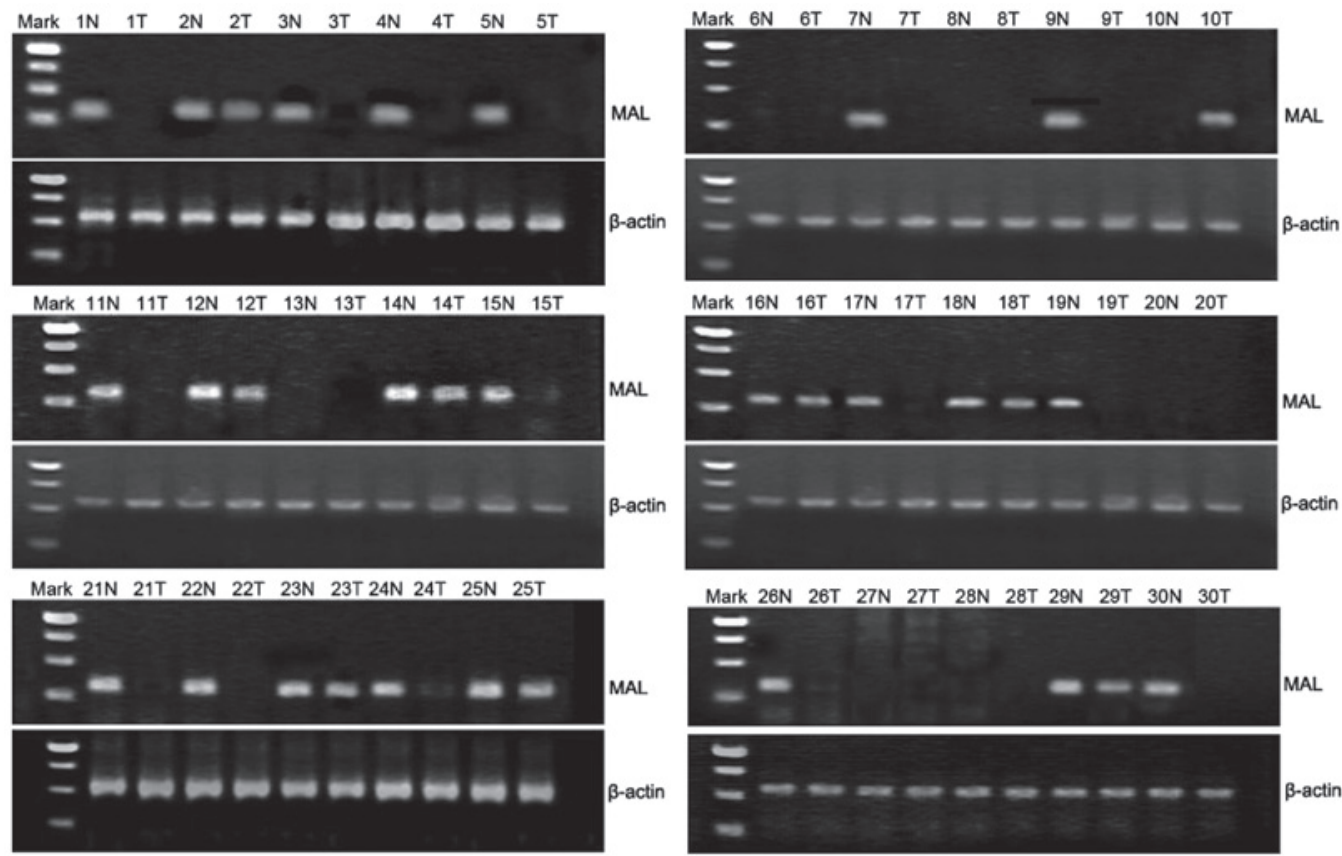

Figure 1. RNA expression of MAL in 30 CRC tissues, as determined by semi-quantitative polymerase chain reaction. $\beta$-actin level was considered as an endogenous control. CRC, colorectal cancer; $\mathrm{N}$, adjacent tissue; T, CRC tissue. MAL, mal, T-cell differentiation protein.
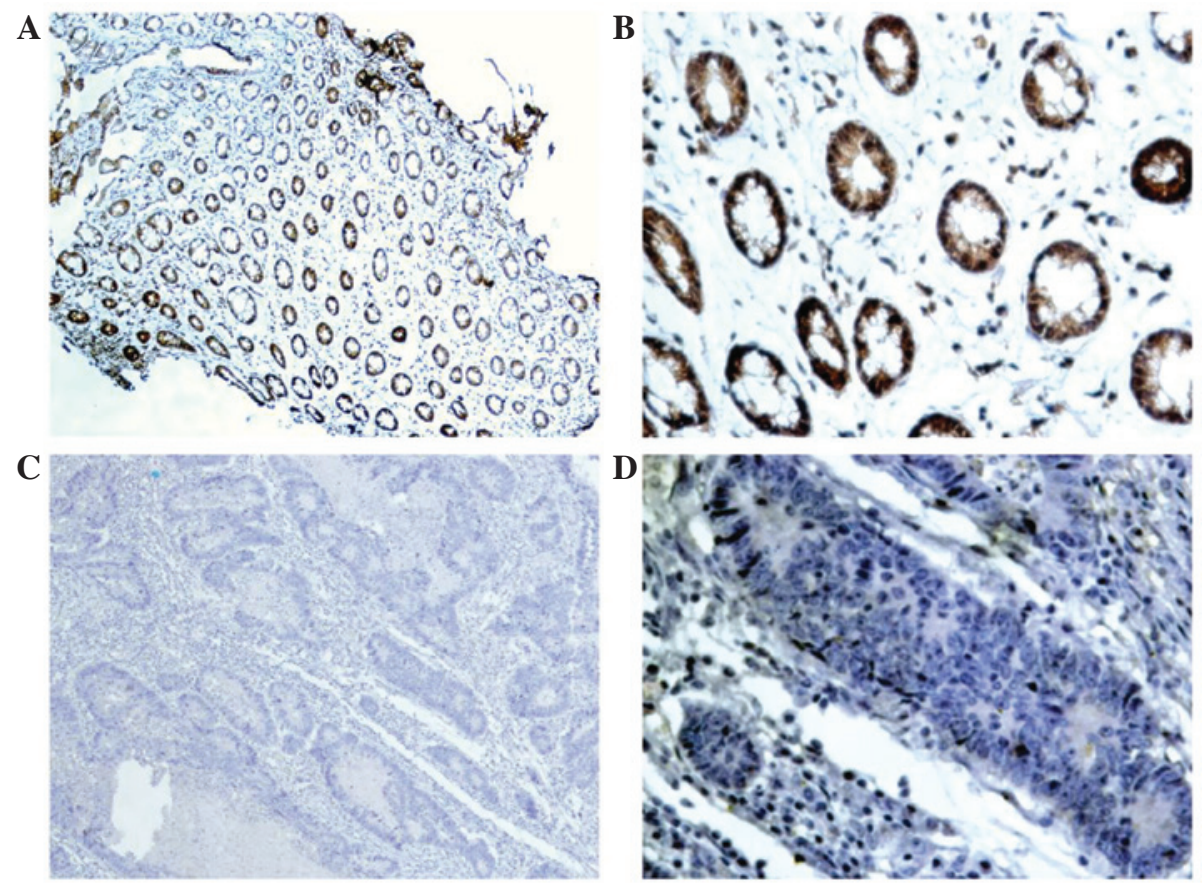

Figure 2. Protein expression of mal, T-cell differentiation protein in CRC tissues, as determined by immunohistochemical analysis. Positive adjacent tissue at (A) x100 and (B) x400 magnification; and negative CRC tissue at (C) x100 and (D) x400 magnification. CRC, colorectal cancer.

rate of $76.7 \%$ (23/30) (Fig. 1). The RNA level of MAL was significantly downregulated in the CRC tissues compared with the adjacent tissues $\left(\chi^{2}=13.125 ; \mathrm{P}=0.001\right)$.

Protein expression level of MAL in CRC and adjacent tissues. By performing immunohistochemical staining, MAL protein was detected in the nucleus and cytoplasm of the CRC tumor cells (Fig. 2). Immunohistochemical staining results showed that 20\% (6/30) of the CRC specimens expressed MAL, while
$66.7 \%$ (20/30) of the adjacent tissues expressed MAL (Table II). The protein level of MAL was significantly downregulated in the CRC tissues compared with the adjacent tissues $\left(\chi^{2}=13.303\right.$; $\mathrm{P}=0.001$ ). According to Spearman's rank correlation test, the RNA level of MAL was significantly positively correlated with the protein level of MAL ( $\mathrm{rs}=0.818 ; \mathrm{P}=0.0001)$.

Correlation between MAL and pathological staging, metastasis status and clinical characteristics of the CRC patients. On the 
Table II. Correlation between MAL protein level and metastatic status in CRC patients.

\begin{tabular}{lcccc}
\hline & & \multicolumn{2}{c}{ MAL expression } & \\
\cline { 3 - 5 } TNM stage & Cases, $\mathrm{n}$ & $(+), \mathrm{n}$ & $(-), \mathrm{n}$ & $\chi^{2}$ \\
\hline CRC with metastases & 12 & 0 & 12 & 5.000 \\
CRC without metastases & 18 & 6 & 12 & 0.031 \\
\hline
\end{tabular}

TNM, tumor-node-metastasis; MAL, mal, T-cell differentiation protein; CRC, colorectal cancer.

Table III. Correlation between MAL protein level and different TNM stage in the colorectal cancer patients.

\begin{tabular}{lcccr}
\hline & & \multicolumn{2}{c}{ MAL expression } & \\
\cline { 3 - 5 } TNM stage & Cases, $\mathrm{n}$ & $(+), \mathrm{n}$ & $(-), \mathrm{n}$ & $\chi^{2}$ \\
\hline I,II & 17 & 6 & 11 & 5.735 \\
III & 13 & 0 & 13 & 0.021 \\
\hline
\end{tabular}

TNM, tumor-node-metastasis; MAL, mal, T-cell differentiation protein.

Table IV. Correlation between MAL protein level and pathological factors in the colorectal cancer patients.

\begin{tabular}{|c|c|c|c|c|c|}
\hline \multirow[b]{2}{*}{ Pathological factors } & \multirow[b]{2}{*}{ Cases, $\mathrm{n}$} & \multicolumn{2}{|c|}{ MAL expression } & \multirow[b]{2}{*}{$\chi^{2}$} & \multirow[b]{2}{*}{ P-value } \\
\hline & & $(+), \mathrm{n}$ & $(-), \mathrm{n}$ & & \\
\hline Tumor location & 30 & 6 & 24 & 1.818 & 0.403 \\
\hline Right colon & 10 & 2 & 8 & & \\
\hline Left colon & 9 & 3 & 6 & & \\
\hline Rectum & 11 & 1 & 10 & & \\
\hline Histological type & & & & 0.833 & N/A \\
\hline Adenocarcinoma & 27 & 6 & 21 & & \\
\hline Mucinous adenocarcinoma & 3 & 0 & 3 & & \\
\hline Differentiation status & & & & 1.429 & 0.490 \\
\hline Highly-differentiated & 6 & 2 & 4 & & \\
\hline Moderately-differentiated & 21 & 4 & 17 & & \\
\hline Poorly-differentiated & 3 & 0 & 3 & & \\
\hline
\end{tabular}

MAL, mal, T-cell differentiation protein; N/A, non-applicable.

Table V. Correlation between MAL protein level and clinical characteristics in the colorectal cancer patients.

\begin{tabular}{|c|c|c|c|c|c|}
\hline \multirow[b]{2}{*}{ Clinical characteristics } & \multirow[b]{2}{*}{ Cases, $\mathrm{n}$} & \multicolumn{2}{|c|}{ MAL expression } & \multirow[b]{2}{*}{$\chi^{2}$} & \multirow[b]{2}{*}{ P-value } \\
\hline & & $(+), \mathrm{n}$ & $(-), \mathrm{n}$ & & \\
\hline Age, years & 30 & 6 & 24 & 1.500 & 0.553 \\
\hline$<60$ & 5 & 0 & 5 & & \\
\hline$\geq 60$ & 25 & 6 & 19 & & \\
\hline Gender & & & & 0.139 & N/A \\
\hline Male & 18 & 4 & 14 & & \\
\hline Female & 12 & 2 & 10 & & \\
\hline
\end{tabular}

MAL, mal, T-cell differentiation protein; N/A, non-applicable. 
basis of the overall evaluation of the immunohistochemical staining score, the present study investigated whether MAL protein expression level was correlated with pathological staging, metastasis status and the clinical characteristics of the CRC patients. As shown in Table III, statistical analysis indicated that the level of MAL expression was significantly lower in CRC tumor-node-metastasis (TNM) stage III than in TNM stages I and II $\left(\chi^{2}=5.735 ; \mathrm{P}=0.021\right)$. According to the analysis of lymph node metastasis, the expression level of MAL was significantly lower in the cases of CRCs with lymph node metastasis compared with those without lymph node metastasis $\left(\chi^{2}=5.000\right.$; $\mathrm{P}=0.031$; Table II). However, the expression of MAL showed no significant correlation with the other pathological factors of the CRC patients, including tumor location, histological type and differentiation status (Table IV), and the clinical characteristics of the CRC patients, including age and gender (Table V).

\section{Discussion}

The formation of polarity in epithelial cells depends on the rigorous maintenance of the regulation of transport and sorting process, which guarantees precise delivery of biosynthetic cargo to varying areas of the plasma membrane (13). The polarized transport of lipids and proteins to the plasma membrane is vital for the functions of the epithelial cells. MAL has been found to be a pivotal component of the machinery for the direct transport route. Loss of MAL leads to the loss of the polarized phenotype that frequently accompanies the neoplastic transformation process (14). Recent studies have suggested that downregulation of MAL has been associated with a variety of human epithelial malignancies, including esophageal (7), breast (8), ovarian (9) and cervical (15) cancers. For example, Mimori et al showed that overexpression of MAL in esophageal tumors exhibited decreased cellular motility, a $\mathrm{G}_{1} / \mathrm{S}$ transition block and increased levels of apoptosis via the Fas signaling pathway (7). Overmeer et al (9) further demonstrated the repression of MAL tumor suppressor activity by promoter methylation during cervical carcinogenesis, providing a predictive biomarker for underlying high-grade lesions in cervical cancer. Beder et al (10) further identified potential genetic and epigenetic mechanisms associated with the downregulation of MAL in head and neck squamous cell carcinomas, including loss of heterozygosity, mutation and hypermethylation. Buffart et al (11) indicated that MAL promoter hypermethylation can be used as a novel prognostic marker in gastric cancer.

For the first time, the present study evaluated the expression of MAL in CRC. RNA and protein expression levels of MAL were analyzed in 30 CRC patients and found to be significantly downregulated in the CRC tissues compared with the adjacent tissue, which was consistent with the previous studies in other epithelial malignancies. To determine the clinical significance of MAL in CRC, the correlation between its expression level and pathological staging, metastasis status and the clinical characteristics of the CRC patients was inves- tigated. The results showed that the expression levels of MAL were significantly associated with different TNM stages and lymph node metastasis, but not with age, gender, tumor site, differentiation status or pathological type. These results indicate that MAL has a putative anti-metastasis function in CRC, and that detection of MAL expression level may be a potential biomarker for malignant CRC.

\section{Acknowledgements}

This study was supported by the Hunan Social Development Support Program (grant number, 2012SK3190) and the Hunan Graduate student research innovation project (grant number, CX2012B090).

\section{References}

1. Jemal A, Siegel R, Ward E, Murray T, Xu J and Thun MJ: Cancer statistics, 2007. CA Cancer J Clin 57: 43-66, 2007.

2. Sung JJ, Lau JY, Goh KL and Leung WK; Asia Pacific Working Group on Colorectal Cancer: Increasing incidence of colorectal cancer in Asia: Implications for screening. Lancet Oncol 6: 871-876, 2005.

3. Ji BT, Devesa SS, Chow WH, Jin F and Gao YT: Colorectal cancer incidence trends by subsite in urban Shanghai, 1972-1994. Cancer Epidemiol Biomarkers Prev 7: 661-666, 1998.

4. Gupta GP and Massagué J: Cancer metastasis: Building a framework. Cell 127: 679-695, 2006.

5. Zhou Y, Wan G, Spizzo R, et al: miR-203 induces oxaliplatin resistance in colorectal cancer cells by negatively regulating ATM kinase. Mol Oncol 8: 83-92, 2014.

6. Alonso MA and Weissman SM: cDNA cloning and sequence of MAL, a hydrophobic protein associated with human T-cell differentiation. Proc Natl Acad Sci USA 84: 1997-2001, 1987.

7. Mimori K, Shiraishi T, Mashino K, et al: MAL gene expression in esophageal cancer suppresses motility, invasion and tumorigenicity and enhances apoptosis through the Fas pathway. Oncogene 22: 3463-3471, 2003.

8. Horne HN, Lee PS, Murphy SK, Alonso MA and Olson JA Jr and Marks JR: Inactivation of the MAL gene in breast cancer is a common event that predicts benefit from adjuvant chemotherapy. Mol Cancer Res 7: 199-209, 2009.

9. Overmeer RM, Henken FE, Bierkens M, et al: Repression of MAL tumour suppressor activity by promoter methylation during cervical carcinogenesis. J Pathol 219: 327-336, 2009.

10. Beder LB, Gunduz M, Hotomi M, et al: T-lymphocyte maturation-associated protein gene as a candidate metastasis suppressor for head and neck squamous cell carcinomas. Cancer Sci 100: 873-880, 2009.

11. Buffart TE, Overmeer RM, Steenbergen RD, et al: MAL promoter hypermethylation as a novel prognostic marker in gastric cancer. Br J Cancer 99: 1802-1807, 2008.

12. Edge SB and Compton CC: The American Joint Committee on Cancer: The 7th edition of the AJCC cancer staging manual and the future of TNM. Ann Surg Oncol 17: 1471-1474, 2010.

13. Schuck S and Simons K: Polarized sorting in epithelial cells: raft clustering and the biogenesis of the apical membrane. J Cell Sci 117: 5955-5964, 2004.

14. Marazuela M and Alonso MA: Expression of MAL and MAL2, two elements of the protein machinery for raft-mediated transport, in normal and neoplastic human tissue. Histol Histopathol 19: 925-933, 2004.

15. Lee PS, Teaberry VS, Bland AE, et al: Elevated MAL expression is accompanied by promoter hypomethylation and platinum resistance in epithelial ovarian cancer. Int J Cancer 126: 1378-1389, 2010. 